\title{
El Género Y El Consumo Hedonista En Saltillo, Coahuila
}

\author{
Mtra. Edith Reyes Ruiz \\ Dra. Adriana Méndez Wong \\ Dr. Jesús Francisco Mellado Siller \\ Universidad Autónoma de Coahuila, Mexico \\ Dra. Laura Estela Fischer de la Vega \\ Universidad Autónoma de México
}

doi: 10.19044/esj.2017.v13n23p1 URL:http://dx.doi.org/10.19044/esj.2017.v13n23p1

\begin{abstract}
In the marketing context, it is essential to know the market. That is why there is a necessity to investigate its behavior in regards to clothes purchase. The aim of this study is to develop a model from the conceptual perspective of the shopper typology scale (Chengedzai, Manillall \& Lawrence, 2014) in its factor: Hedonist consumer. The study included 250 adult participants $(59.2 \%$ women and $40.8 \%$ men) living in the City of Saltillo, Coahuila, Mexico. A discriminate analysis was performed to get a membership model of the gender of the variables of Hedonist trend consumer. The results of the findings show meaningful differences between men and women. Women enjoy more shopping, and they have fun while doing it. They also spend more time in this activity.
\end{abstract}

Keywords: Shopping behavior, Hedonist consumer, gender, shopper typology

\section{Resumen}

En el ámbito de la Mercadotecnia es imprescindible conocer al mercado, por lo cual existe una necesidad de investigar su comportamiento en cuestión de compra de ropa. El presente estudio tuvo como objetivo desarrollar un modelo desde la perspectiva conceptual de la escala Tipo de consumidores de ropa de Chengedzai, Manillall y Lawrence (2014) en su factor: Consumidor Hedonista. El estudio incluyó a 250 participantes adultos residentes en la Ciudad de Saltillo, Coahuila, México. 59.2\% mujeres y $40.8 \%$ hombres; se realiza un análisis discriminante para obtener un modelo de pertenencia de Género de las variables de consumidor de moda Hedonista, encontrando resultados que muestran que existen diferencias significativas 
en los hombres y mujeres: las mujeres disfrutan más el comprar la ropa, se divierten mientras lo hacen y dedican mas tiempo a dicha actividad.

Palabras Claves: Comportamiento de Compra, Consumidor Hedonista, Género, Tipos de Consumidores

\section{Introducción}

Desde el punto de vista de la mercadotecnia, entender el comportamiento de compra de los consumidores no es tarea fácil. Segun Philip Kotler, los factores básicos que influyen en la conducta de los consumidores son de dos tipos externos e internos, entre los primeros destacan la cultura, los valores, los aspectos demográficos, el status social, los grupos de referencia, y el hogar; entre los segundos están las necesidades, los deseos, la personalidad, las emociones, las actitudes, los motivos, las percepciones y el aprendizaje. Ambos tipos (externo e interno) no están separados, por el contrario se entrelazan para definir el comportamiento del individuo en el momento de elegir un determinado producto (Kotler, 2007). Entre los consumidores existen aquellos que encuentran la actividad de comprar disfrutable y compran solo por diversión, son los denominados consumidores hedonistas.

\section{Revisión de literatura}

O‘ Shaughenessy (2002) señala que el hedonismo promueve el placer como el único objeto del deseo y que las motivaciones se basan en la búsqueda incesante de la autogratificación y en el beneficio de la dimensión egoísta del ser humano.

Featherstone (1991) citado por Muñoz (Muñoz Molina, 2004), en su texto Cultura de consumo y posmodernidad, identifica dos aspectos fundamentales en la sociedad de consumo actual: por un lado encuentra que los patrones de consumo son una fuente de diferenciación y de estatus $\mathrm{y}$, por otro, se evidencia que el consumo es una fuente de fantasía y placer en un universo de estímulos permanentes.

Los conceptos de género y consumo, aparecen ya relacionados en lo que se puede denominar la obra clásica de la sociología del consumo "La teoría de la clase ociosa". Veblen a finales del siglo XIX señala cómo el consumo es un ámbito femenino, ya que es a través del mismo desde donde la mujer expresa el estatus social del marido, en lo que se ha denominado un consumo vicario, un consumo de representación social del estatus del cabeza de familia. En una misma línea, el autor destaca cómo la indumentaria femenina no hace otra cosa que separar a la mujer de la esfera productiva; los corsés, los zapatos de tacón, los sombreros etc. son objetos que imposibilitan 
la realización de cualquier actividad útil, separando, por tanto, a la mujer del espacio productivo (Castillo, 1988) citado por (Borrás Catalá, 2007).

Alba Carosio (2008) manifiesta que en la estrategia de creación de mercados se desarrolla una nueva manera de ver a las mujeres, una nueva óptica: la mujer cuidadora del hogar, madre y gestionadora de lo doméstico, se transforma en mujer consumidora. Porque para manejar el hogar las mujeres deben comprar insumos y ello las convierte en el "objetivo" más apetecido de las estrategias de mercadeo. Aunque las mujeres siempre cuidaron el hogar y sus miembros, en la propuesta de la sociedad de consumo la actividad del ama de casa de comprar es central. Desde este punto de vista, las "decisiones de compra" deben ser inteligentes, para que pueda lograrse el bienestar de los miembros de la familia, y esta responsabilidad recae sobre las mujeres. Las mujeres comienzan a ser vistas como las líderes indiscutibles en consumo, puesto que $80 \%$ de las decisiones de compra del hogar las toman las mujeres, son negocio seguro. Dicen los expertos en marketing que "son mejores compradoras que los hombres".

En los ultimos años en Barcelona, se ha visto la entrada masculina en la compra de alimentos, se ha venido dando desde los consumos que reportan más placer que obligación, carga o responsabilidad, es decir los hombres suelen acompañar a las mujeres a realizar la compra de alimentos y bebidas, pero ellos realizan o toman la decisión de compra de determinados productos de lujo alimentario o de determinadas exquisiteces. Mientras son ellas las que realizan y gestionan la compra semanal de los alimentos y bebidas de tipo más cotidiano y usual. Otro factor explicativo de la entrada de los hombres en el consumo, son los nuevos valores que marcan las sociedades actuales de consumo, también llamadas postmodernas: el placer o la búsqueda del mismo como finalidad, es decir el Hedonismo, ha penetrado en la mentalidad de los consumidores con gran fuerza. Y es precisamente este el que representa uno de los canales de entrada de los hombres en la realización de estos consumos vinculados a la compra especial de alimentos y bebidas (Borrás Catalá, 2007).

Respecto al interés del tema, en el contexto científico, existen trabajos previos que analizan el comportamiento de compra de ropa:

Sproles y Kendall (1986) conceptualizan ocho características básicas de estos estilos y desarrollan un inventario de estilos de consumo para medirlas empíricamente. Llevan a cabo la validación mediante un Análisis factorial. Los resultados de la investigación se discuten y se sugieren aplicaciones del inventario de estilos de consumo en educación del consumidor, investigación del consumidor y asesoramiento financiero familiar.

Las dimensiones y perfiles de estilos de toma de decisiones en el consumo de jóvenes y adultos en China, se investigan mediante un modelo 
de modificación de estilos de toma de decisiones del consumidor y los datos fueron obtenidos en cinco universidades chinas. Los resultados se comparan luego con los de estudios similares utilizando los resultados de estudios norteamericanos y coreanos. Los resultados arrojan que las dimensiones de estilos de toma de decisiones de consumo son similares en estos tres países (Fan \& Xiao, 1998).

Para Cathy Bakewell y Vincent-Wayne Mitchell, los factores ambientales influyen de manera significativa en los compradores de la Generación Y. Realizaron un estudio en el 2003 donde examinan la toma de decisiones de los consumidores utilizando la escala Consumo Inventario de Estilos (CSI) propuesta por Sproles y de Kendall (1986). Dicho estudio utiliza la CSI como base para la segmentación de generación Y, discutiendo las implicaciones de los resultados para los minoristas y los profesionales de marketing que atienden a los consumidores de la Generación Y (Bakewell \& Mitchell, 2003).

Chengedzai , Manillall, y Lawrence (2014), realizan una investigacion donde su objetivo principal fue examinar las tipologías de compradores de la Generación Y, considerando las variables edad y moda de ropa. Utilizaron como instrumento una encuesta y su muestra fue de 230 sujetos pertenecientes a la Generación Y. Utilizaron el Análisis factorial exploratorio para identificar los tipos de compradores. Y aplicaron la prueba de Kruskal-Wallis para examinar la influencia de la edad sobre las tipologías de compradores identificados. El estudio identificó siete tipologías de compradores que son aplicables a los millenials: Consciente de la moda, Hedonista, Consciente de la marca, innovador, Consciente de la calidad, Indecisos, Lealtad a la marca. El valor del estudio es que estos resultados pueden permitir a los vendedores a comprender y predecir el comportamiento de compra de los consumidores de la generación Y, facilitando el desarrollo e implementación de estrategias de marketing más eficaces.

\section{Metodología}

Se realizó un estudio cuantitativo-explicativo transversal y un análisis discriminante para obtener un modelo de pertenencia de Género de las variables de Consumidor Hedonista de Ropa, en la Ciudad de Saltillo, Coahuila, desde la perspectiva conceptual de la escala Tipo de consumidores de ropa de Chengedzai, Manillall y Lawrence (2014) en su factor: Consumidor Hedonista. 


\section{Muestra}

La investigación, utilizó una muestra de 250 participantes residentes en la ciudad de Saltillo, Coahuila, correspondiendo un 59.2\% mujeres y $40.8 \%$ hombres.

METODOLOGÍA (Hernández, Fernández, \& Baptista, 2014)

Estadísticas de fiabilidad

Alfa de Cronbach $\mathrm{N}$ de elementos

\begin{tabular}{l|l}
\hline .790 & 8 \\
\hline
\end{tabular}

\section{Instrumento de medición}

Para el presente estudio se utiliza la escala desarrollada por Chengedzai, Manillall y Lawrence (2014) y está compuesto de siete variables:

1. Consciente de la moda: Característica representativa de un consumidor quien está motivado a mantenerse al día con tendencia de moda y estilos.

2. Hedonista: Característica para medir el grado en donde un consumidor encuentra la actividad de comprar disfrutable y compra solo por diversión.

3. Consciente de la marca: Mide la orientación de un consumidor para lo más caro y de marcas reconocidas.

4. Innovador: Identifica consumidores que gustan de productos nuevos e innovadores y se emocionan buscando nuevos productos.

5. Consciente de la calidad: Característica que mide el grado en el que un consumidor busca cuidadosa y sistemáticamente la mejor calidad en productos.

6. Indecisos: Identifica al consumidor que percibe muchas marcas y tiendas de donde escoger, así como información.

7. Lealtad a la marca: Indica al consumidor que tiene marcas y tiendas favoritas y que han formado hábitos cerca de ellas.

Los Ítems del Constructo Hedonista son:

1. Comprar ropa no es una actividad placentera.

2. Ir a comprar ropa es una de las actividades que más disfruto en mi vida.

3. Disfruto comprar por diversión.

4. Hago mis compras rápidamente.

5. No pierdo tiempo solo comprando.

6. Comprar ropa es tiempo perdido.

7. Si vale mi tiempo al comprar en las tiendas.

8. Comprar ropa satisface mi sentido de curiosidad.

El cuestionario se diseñó con una escala 1 a 5 tipo Likert, en donde 5 significa "totalmente de acuerdo" y 1 "totalmente en desacuerdo". 


\section{Hipótesis} género".

Hi "Las variables de Consumidor Hedonista presentan diferencia por

\section{Resultados}

Se realiza un análisis discriminante para obtener un modelo de pertenencia de género de las variables de consumidor de moda Hedonista encontrándose los siguientes resultados (Nunnally, 1987).

Tabla 1. Resumen de procesamiento de casos de análisis

Casos sin ponderar

\begin{tabular}{ccc|c} 
& Casos sin ponderar & $\mathrm{N}$ & Porcentaje \\
\hline Excluido & 250 & 100.0 \\
\hline & $\begin{array}{c}\text { Códigos de grupo perdidos o fuera de } \\
\text { rango }\end{array}$ & 0 & .0 \\
\cline { 2 - 3 } & $\begin{array}{c}\text { Como mínimo, falta una variable } \\
\text { discriminatoria }\end{array}$ & 0 & .0 \\
\cline { 2 - 3 } & $\begin{array}{l}\text { Faltan ambos códigos de grupo, los } \\
\text { perdidos o los que están fuera de rango } \\
\text { y, como mínimo, una variable } \\
\text { discriminatoria }\end{array}$ & 0 & .0 \\
\hline$\quad$ Total & 0 & 100.0 \\
\hline
\end{tabular}

Fuente: Elaboración Propia

La tabla demuestra que no hay datos incompletos y datos fuera de rango, lo que Indica que la base de datos no presenta casos vacíos ni fuera de rango, es decir la base es correcta para la aplicación de Análisis Discriminante (Moreno, 2005).

\section{Análisis 1. Resumen de Funciones Discriminantes Canónicas}

Tabla 2. Autovalores

\begin{tabular}{cc|c|c|c} 
Función & Autovalor & $\%$ de varianza & $\%$ acumulado & $\begin{array}{c}\text { Correlación } \\
\text { canónica }\end{array}$ \\
\hline 1 & $.065^{\text {a }}$ & 100.0 & 100.0 & .246 \\
\hline
\end{tabular}

Se utilizaron las primeras 1 funciones discriminantes canónicas en el análisis.

Fuente: Elaboración propia

Tabla 3. Lambda de Wilks

\begin{tabular}{cc|c|c|c} 
Prueba de funciones & Lambda de Wilks & Chi-cuadrado & gl & Sig. \\
\hline 1 & .139 & 15.253 & 8 & .044 \\
\hline
\end{tabular}

Fuente: Obtenida de resultados de SPSS

En la tabla 3 se observa que la suma de cuadrados intra-grupos, sobre la variación dentro de grupos, se interpreta junto con el estadístico Lambda de Wilks, en este caso al autovalor está cercano a cero pero la correlación 
canónica es baja lo que Indica que las variables no permiten distinguir bien los dos grupos; pero el Lambda de Wilks, prueba Hipótesis nula de Centroides igual a cero, y éste valor se encuentra cercano a cero, lo que señala que se rechaza $\mathrm{H} 0$ lo que muestra que hay diferencia entre los grupos, debido a la significancia de $\mathrm{P}<$ que 0.05 . También, se considera el Lambda transformado que se expresa como valor de Ji cuadrado de Schatzoof 1966 (Hair, E., Tatham, \& Black, 1999) que tiene un valor de 15.253, y un alfa de 0.44 lo que rechaza hipótesis de igualdad de medias de centroides, confirmando con ésto la diferencia entre grupos.

Tabla 4. Funciones en centroides de grupo

\begin{tabular}{cc} 
& Función \\
Sexo Género & 1 \\
\hline 1 Hombre & .265 \\
\hline 2 Mujer & -.241 \\
\hline
\end{tabular}

Las funciones discriminantes canónicas sin estandarizar se han evaluado en medias de grupos.

Fuente: Elaboración Propia

La tabla 4 señala que para el grupo de hombres las funciones en promedio de centroides es de tipo positivo, y las funciones para mujeres son de tipo negativo, esto servirá para determinar junto con los coeficientes de la función discriminante canónica estandarizados la pertenencia de las variables a los grupos de hombre y mujer.

Tabla 5. Coeficientes de función discriminante canónica estandarizados

\begin{tabular}{cc} 
& Función \\
& 1 \\
\hline V11 B1 Actividad placentera: comprar ropa & -.240 \\
\hline V12 B2 Disfrutar de la vida comprando ropa & -.124 \\
\hline V13 B3 Disfruta comprar por diversión. & -.310 \\
\hline V14 B4 Realiza compras rápidamente & .963 \\
\hline V15 B5 No pierdo tiempo solo comprando & -.468 \\
\hline V16 B6 Comprar ropa es pérdida de tiempo & .083 \\
\hline V17 B7 Valora el tiempo comprando & -.027 \\
\hline V18 B8 Satisfacción de curiosidad comprando ropa & .432 \\
\hline
\end{tabular}

Fuente: Elaboración propia

El examen de los coeficientes de función discriminante canónica de coeficientes estandarizados, que se encuentran en la tabla 5, utiliza este tipo de valores ya que las variables pueden tener una métrica diferente, y los valores estandarizados iguala la métrica. muestra las variables de: realiza compras rápidamente con un coeficiente de .963 ; satisfacción de curiosidad comprando ropa con un coeficiente de .432 y considerar que comprar es pérdida de tiempo con un valor de .083 son variables que por su signo pertenecen al grupo sexual masculino, y el valor positivo señala que un incremento en la media de esas variables en un sujeto es característica para 
pertenecer al grupo de hombres; en contraposición los resultados de las variables que obtuvieron signos negativos en el $\mathrm{AD}$, y que caracterizan a las mujeres son: no pierdo el tiempo comprando con un valor de -.468, disfruta comprar por diversión con una cantidad de -.310; la compra de ropa es actividad placentera con -.240; disfrutan la vida comprando ropa con -.124; y valoran el tiempo cuando compran con -.0.27. por lo mismo un valor negativo implica un valor por encima de la media tiende como efecto un decremento que hará disminuir la puntuación discriminante y como consecuencia el sujeto pertenece a la caracterización del sexo femenino. Todo lo anterior nos señala que los hombres compradores de ropa hedonista presentan las siguientes características: no pierden el tiempo comprando ropa, son curiosos para la ropa, sin embargo las compras que realizan son hechas en espacios cortos de tiempo. Para las variables identificadoras de sexo femenino son: ellas disfrutan el comprar ropa, lo que les asegura diversión, y les permite valorar el tiempo en sus compras por lo mismo para ellas esto es disfrutar el tiempo.

\section{Discusión}

En las primeras líneas de este trabajo se justifica el interés por el estudio del comportamiento de compra hedonista específicamente de ropa. Hay que añadir, además la escasez de trabajos empíricos existentes en México, lo que destaca la pertinencia de estudios como éste.

En concreto, el estadístico Lamba de Wilks nos señala que se rechaza la $\mathrm{H} 0$ pues se muestra que hay diferencia entre hombres y mujeres consumidores hedonistas, coincidiendo con Alba Carosio (Carosio, 2008) quien sustenta que en la sociedad actual las mujeres son vistas como las líderes indiscutibles en consumo,

Se coincide con Borras (Borrás Catalá, 2007) quien afirma que el placer o la búsqueda del mismo como finalidad, es decir el Hedonismo, ha penetrado en la mentalidad de los consumidores con gran fuerza.

La relevancia del presente estudio es la Aportación con evidencia empírica de la validación de un modelo propuesto y con ello, seguir avanzando en la creación de un mayor cuerpo de conocimientos alrededor del área de mercadotecnia.

\section{Conclusión}

Los resultados obtenidos al realizar un análisis discriminante para obtener un modelo de pertenencia de género de las variables de consumidor de moda Hedonista, ofrecen un primer diagnóstico: sí existen diferencias significativas en los hombres y mujeres, pues las mujeres disfrutan más el comprar la ropa, se divierten mientras lo hacen y dedican mas tiempo a dicha actividad. Mientras que los hombres realizan compras rápidamente, 
encuentran satisfacción de curiosidad comprando ropa y la mayoria considera que comprar es pérdida de tiempo.

En contraste con el estudio de Chengedzai, Manillall, y Lawrence (2014), entre los consumidores milenials, que no hace diferencias con la variable género, y generaliza sus resultados entre ambos sexos. Dichos resultados muestran que los entrevistados en general, hombres y mujeres, encuentran que la compra de ropa de moda es una actividad agradable y placentera. La concordancia con dicho estudio es en la definición que tienen los constructos acerca del consumidor hedonista: los consumidores hedonistas no sienten que ir de compras sea una pérdida de tiempo; además se emocionan por la tarea de comprar y por comprar algo novedoso. Los consumidores hedonistas son asociados con los rasgos de compra consciente recreativo y están de acuerdo en que comprar ropa es una de las actividades disfrutables en sus vidas, que se divierten comprando, que comprar ropa satisface su sentido de curiosidad y que comprar es una aventura para ellos.

En otro renglón, se reconoce como limitante del estudio actual, en que los hallazgos están restringidos al mercado de prendas de vestir de moda y en un espacio específico, la ciudad de Saltillo, Coahuila en México. Se visualizan nuevas líneas de investigación a partir de los resultados obtenidos en el presente estudio, ampliando la muestra a otros estados de México, a fin de realizar análisis comparativos, así mismo contemplando incluir otras variables de estudio como lo es, la edad del entrevistado.

\section{References:}

1. Bakewell, C., \& Mitchell, V.-W. (2003). "Generation Y female consumer decision-making styles". International Journal of Retail \& Distribution Management, 31(2), 95-106.

2. Borrás Catalá, V. (2007). Inequalities in consumption through. Revista Española de Sociología, 139-156.

3. Carosio, A. (2008). Revista de estudios de género. La ventana. Recuperado el 2017, de El género del consumo en la sociedad de consumo.:

http://www.scielo.org.mx/scielo.php?script=sci_arttext\&pid=S140594362008000100006\&lng=es\&tlng=es.

4. Chengedzai , M., Manillall , D., \& Lawrence, M. (2 de Abril de 2014). 'Shopper typologies amongst a Generation Y consumercohort and variations in terms of age in the fashionapparel market'. Recuperado el 26 de Enero de 2015, de http://www.actacommercii.co.za: http://dx.doi.org/10.4102/ac.v14i1.209

5. Fan, J. X., \& Xiao, J. J. (1998). Consumer decision-making styles of young-adult Chinese. . Journal of Consumer Affairs, , 275-294. 
6. Hair, J. F., E., A. R., Tatham, R. L., \& Black, W. C. (1999). Análisis Multivariante (quinta Edición). Madrid, España.: Pearson Educación.

7. Hernández, S. R., Fernández, C. C., \& Baptista, L. P. (2014). Metodología de la Investigación (Sexta Edición). México: McGrawHill.

8. Kotler, P. A. (2007). Marketing. Versión para Latinoamérica. Mexico: Pearson.

9. Moreno, O. J. (2005). Analisis Multivariante en Investigaciones de Calidad del Servicio. Revista Venezolana de Analisis de Coyontura, 275-308.

10. Muñoz Molina, Y. (2004). La satisfacción del consumidor en las experiencias hedonistas. Academia. Revista Latinoamericana de Administración, 52-67.

11. Nunnally, J. C. (1987). Teoría Psicométrica. México: Ed. Trillas .

12. O'Shaughenessy, J., \& O'Shaughenenessy, N. (2002). "Marketing, the Consumer Society and Hedonism". European Journal of Marketing, 36, 524-548.

13. Sprotles, G. B., \& Kendall, E. L. (1986). A Methodology for Profiling Consumers' Decision-Making Styles. Journal of Consumer Affairs, 267-279. 